\title{
Civilisations
}

Revue internationale d'anthropologie et de sciences

humaines

59-1 | 2010

Sexualités : apprentissage et performance

\section{Ritualisation mémorielle et construction ethnique postcommuniste chez les Hongrois de Transcarpathie (Ukraine)}

\section{Anne-Marie Losonczy}

\section{OpenEdition}

\section{Journals}

Édition électronique

URL : http://journals.openedition.org/civilisations/2248

DOI : 10.4000/civilisations. 2248

ISSN : 2032-0442

\section{Éditeur}

Institut de sociologie de l'Université Libre de Bruxelles

\section{Édition imprimée}

Date de publication : 28 juin 2010

Pagination : 131-150

ISBN : 2-87263-029-5

ISSN : 0009-8140

\section{Référence électronique}

Anne-Marie Losonczy, «Ritualisation mémorielle et construction ethnique postcommuniste chez les Hongrois de Transcarpathie (Ukraine) », Civilisations [En ligne], 59-1 | 2010, mis en ligne le 29 juin 2013, consulté le 21 avril 2019. URL : http://journals.openedition.org/civilisations/2248 ; DOI : 10.4000/ civilisations. 2248 


\title{
Ritualisation mémorielle et construction ethnique postcommuniste chez les Hongrois de Transcarpathie (Ukraine)
}

\author{
Anne-Marie LOSONCZY
}

Notre passé est imprévisible

Joseph Brodsky

Résumé : Issu d'une recherche ethnographique, entamée sur le terrain en 2008, ce texte propose d'interroger dans le contexte post-communiste de la région frontalière de Transcarpathie (Ukraine), un double rapport. D'abord, celui existant entre la construction publique d'une mémoire collective victimaire liée à la déportation au Goulag et sa ritualisation commémorative périodique d'un côté, et les modes d'énonciation et les non-dits des gloses mémoriels informels dans la société magyarophone rurale et urbaine, (re)construisant ses identifications collectives, de l'autre. Ensuite, ce texte tente de circonscrire le lien paradoxal entre les pratiques et discours mémoriels ethnicisés et le caractère nécessairement muti-ethnique et interactionnel des activités économiques, liées au caractère pluri-frontalier de la région, qui seules assurent la survie et la reproduction des groupes, notamment par la contrebande et l'accueil touristique informel construit autour des lieux de mémoire patrimonialisés.

Mots-clés : mémoire, Transcarpathie, Ukraine, ethnicité, frontière, nationalisme, post-communisme.

\begin{abstract}
Drawing on ethnographical research conducted since 2008 in Transcarpathia (Ukraine), this article raises two main issues regarding memory processes in a post-communist context. The first question bears on the relation between the public construction of a collective memory of victimhood grounded in the Goulag experience, and its ritualization, on the one hand, and the silences and uneases of informal memory glosses in the urban and rural local Magyar-speaking society (re)making its categories of collective identification. The second issue arises from the paradoxical relation between ethnicized memory practices and discourses on the one hand, and the necessarily multi-ethnic and interactional nature of economic activities, grounded in the existence of multiple borders in the region, while some of these economic activities, such as smuggling and informal touristic tours built around the local post-communist lieux de mémoire, are precisely crucial in the reproduction of the local society.
\end{abstract}

Key words: memory, Transcarpathia, Ukraine, ethnicity, boundary, nationalism, post-communism. 


\section{$\mathrm{I}$}

nterroger la notion de mémoire collective (Halbwachs 1994 [1925]) signifie rappeler sa structure feuilletée : au-delà de son usage politique, elle comprend la persistance du passé qui reste imprimé dans le présent, souvent en deçà de la conscience, la perpétuation de pratiques et de représentations dans la définition du groupe, le rappel du passé par les souvenirs matériels et narratifs transmis de génération en génération, la mémoire sans souvenir, ancrée dans la recherche et la reconstruction délibérées d'éléments du passé, enfin des bribes de connaissance du passé, sédimentées dans les consciences, collectivement partagées et souvent irriguées d'affect. Facettes constitutives du processus mémoriel, le silence, l'oblitération et le non-dit autour de certains contenus mémoriels - qui ne se confondent ni avec le secret ni avec l'oubli - constituent souvent, en tant que stratégie sociale historiquement construite et transmise, un marqueur identitaire implicite des sociétés, traçant les contours fluides d'une « intimité culturelle » (Herzfeld 2007 [1997]), productrice d'une sorte de communauté du non-dit. L'irruption d'une mise en scène publique et une explicitation des composantes de cette communauté sont alors de nature à restructurer autant les limites extérieures du groupe que les rapports sociaux en son sein.

Les débats actuels en Europe, parfois très vifs, confrontant histoire et mémoire (Wachtel 1986), tentent de tracer les limites et la légitimité de leurs champs respectifs et s'interrogent sur les conditions de leur construction; ils spécifient, souvent de manière contrastive, les caractéristiques de leurs pratiques et de leurs discours. Selon les historiens, l'historicisation peut se définir par une mise en chronologie, un récit contextuel, causal et appuyé sur des sources documentaires écrites et un relatif détachement, par rapport au présent, des événements considérés. L'approche du champ mémoriel d'une société locale post-communiste offre quelques pistes de réflexion supplémentaires sur les figures d'emboîtement entre ces deux régimes de rapport au passé, de même que sur les processus de transformation et les vaet-vient de certains événements et acteurs entre histoire, mémoire collective, et mémoires individuelles testimoniales.

La fin des régimes communistes en Europe centrale et orientale a entraîné, selon des modalités propres à chaque pays, une crise, une rupture et un changement de légitimité dont l'étendue, au-delà de l'espace politique, juridique et économique, a englobé et bouleversé l'ensemble du corps social (Hann, Sarkany et Skalnik 2005). L'émergence subséquente de nouveaux acteurs sociaux, civils et politiques, s'est accompagnée de la constitution d'élites concurrentes dont la lutte pour la légitimité nationale et internationale ne pouvait reposer que sur le déni de tout rapport de continuité avec l'héritage du communisme. La recherche d'autres passés, qui puissent fonder une généalogie politique et morale nouvelle, a suscité, face au passé communiste récent, deux voies qu'ont empruntées les initiatives politiques : celle de l'oblitération et celle de la construction d'une nouvelle mémoire collective d'événements historicisés auparavant. Cette dernière doit donc opposer à l'historiographie et à la littérature mémorielle officielles produites par le régime communiste, des événements et des figures qui avaient été tus, effacés, délégitimés, exilés dans le silence, le non-dit, le secret et les mémoires privées.

Le démantèlement d'une historicité officielle antérieure par un nouveau régime de mémoire publique, au-delà de ses enjeux de légitimation des nouveaux acteurs politiques du moment, loin de s'y opposer, peut au contraire constituer l'étape intermédiaire à la revendication d'une nouvelle historicité. Le socle de cette revendication est l'appel à la " vérité historique ", 
entendue à la fois comme rétablissement d'un ensemble de faits oblitérés ou déformés et comme Némésis, entité transcendante qui reclasse les acteurs du passé sur l'axe du Bien et du Mal à la lumière du présent. Toutefois la « mise en mémoire » publique, particulièrement d'un passé récent, butte sur la multiplicité des témoins encore vivants, les aléas de leurs positions successives sur l'échiquier politique de la période (Hofer 1992), les conflits entre mémoires sectorielles et leur éparpillement. L'usage politique de la mémoire semble donc exiger la construction et la fixation d'un « bloc de mémoire » unifié qui fait disparaître la multiplicité, les contradictions et les paradoxes des mémoires individuelles ou sectorielles.

Mais la somme récente de souffrances, multiples, enchevêtrées et oblitérées, qui émerge dans l'espace public des sociétés post-communistes, articulée à une exigence d'empathie, emblème et nouvelle figure dominante des sensibilités démocratiques européennes, oriente souvent cette entreprise vers la construction d'une Victime collective, personnification de la Vérité historique, susceptible de devenir objet d'identification émotionnelle et de commémoration rituelle. Ce processus constitue l'une des manifestations de la nature culturelle des mémoires patrimonialisées dans les sociétés contemporaines sécularisées.

Aussi le propos de ce texte est-il de situer les multiples composantes du champ mémoriel de la société magyarophone de la Transcarpathie dans le contexte d'une (re)construction des identifications collectives, d'en saisir les tensions, et les acteurs, les modes et contextes d'énonciation et de ritualisation de contenus mémoriels diversifiés ainsi que leurs interactions et les espaces-temps qui les articulent. Entamée en 2008, la recherche ethnographique dont il est issu, est centrée sur la recomposition des interfaces multi- ethniques, religieuses et économiques transfrontalières en Transcarpathie, à partir de l'étude du monde social magyarophone. Le choix de ce dernier, comme point de départ, s'explique par ma connaissance de la langue hongroise, mais la recherche devra s'ouvrir vers les groupes et espaces sociaux ruthènes, ukrainiens et russes et le processus complexe de leur intégration dans le nouvel espace social ukrainien, dès que mon (ré)apprentissage du russe, langue véhiculaire dans le pays, me le permettra. Les deux premiers séjours se sont déroulés dans la ville de Beregovo, trois villages proches, ainsi que deux villages magyarophones (Chap et Tiszapéterfalva), à proximité immédiate de la frontière hungaro-ukrainienne, avec quelques visites dans les villages à prédominance ruthène proche du col de Verecke dans les Carpathes. Au-delà de l'observation et l'accompagnement de certaines activités économiques (commerce informel, accueil de touristes, agriculture) et de visites familiales, la mémoire de la déportation massive de la population masculine au Goulag et les étapes de sa mise en commémoration publique émergeant constamment et sans question préalable dans les conversations et entretiens avec villageois, ouvriers, enseignants locaux ou commerçants, survivants, descendants ou voisins de déportés, elles ont fini par s'imposer dans ma recherche et élargir mes contacts et mes lectures vers les fondateurs et dirigeants régionaux et locaux des deux organisations politiques hongroises, et vers les récits et recueils de témoignages.

\section{La valse des frontières, des toponymes et du temps : la périphérie en héritage}

L'Europe centrale et orientale d'aujourd'hui est composée d'États multi-ethniques, qui se caractérisent notamment par l'existence sur leurs frontières de groupes ethniques dont la spécificité est qu'ils possèdent des correspondants - de même ethnie et langue - construits en états-nations, en général limitrophes. Dès lors, l'historiographie et l'ethnographie de l'Europe centrale les distinguent par le terme de "minorités nationales ». Ces dernières 
furent créées pour la plupart par des traités de paix achevant les deux guerres mondiales, et résultèrent de multiples déplacements de population et redécoupage des frontières étatiques (Bibo 1986 [1946]), celles-ci séparant souvent politiquement les « minorités » de leur Étatnation « majoritaire ». Il en découle que nombre d'entre elles sont disséminées dans des régions limitrophes des frontières étatiques - historiquement changeantes - et leur sociabilité est fortement marquée par ce qu’on peut appeler un « vécu frontalier $»^{1}$.

Ainsi la minorité nationale constituerait une collectivité ethnique d'un type particulier dont la loyauté politico-juridique (citoyenneté) et la loyauté nationale (ethno-culturelle et référentielle) ne coïncident pas. Les liens politiques définis par l'organisation de l'État (que Thomas Eriksen désigne comme l'aspect «formel » du nationalisme) et le fonctionnement local de la société civile, de même que ses représentations culturelles (le " nationalisme informel »), loin de se compléter comme le plus souvent, se polarisent (Eriksen 1993). Cependant les groupes minoritaires se caractérisent également par un champ politique interne propre, dont certains acteurs sont porteurs de relations avec d'autres groupes équivalents et avec l'État (Hobsbawm 1992), et où des mobilisations identitaires peuvent s'institutionnaliser. Par ailleurs, la solidarité et l'identité ethno-culturelles transfrontalières, qui attachent ces minorités à leur « État-nation », sont mobiles et changeantes.

$\mathrm{Au}$ début du $20^{\mathrm{e}}$ siècle, la région, appelée Transcarpathie aujourd'hui, appartenait aux quatre départements à population multi-ethnique du Nord-Est du Royaume de Hongrie, partie de la monarchie austro-hongroise. Elle ne possédait aucun nom régional propre, ni aucune identité régionale distinctive. En 1910 les statistiques estiment sa population à environ 600.000 personnes, composée à $60 \%$ de Ruthènes, bergers et agriculteurs habitant la zone montagneuse des Carpathes, alors que la plaine de l'amont du fleuve Tisza, ainsi que les bourgs et les villes étaient peuplés d'agriculteurs magyars (25\%), d'une importante communauté juive, en grande partie magyarophone, et d'un nombre réduit de Souabes (germanophones), de Roumains, de Slovaques et de Tziganes, souvent magyarophones (Szabo 1993). La population catégorisée comme «magyare » par les statistiques du Royaume y apparaît déjà démographiquement minoritaire : en revanche, sa langue domine la région et sert de véhicule à tous les échanges inter-ethniques. Les frontières entre les groupes apparaissent poreuses, notamment grâce au commerce et aux intermariages.

En 1918, avant d'être rattachée à la Tchécoslovaquie, la région prend brièvement le nom de Russka Kraina (territoire ruthène) et se constitue en province autonome au sein du royaume de Hongrie, à la demande de l'élite ruthène naissante. À l'issue du traité de Trianon, elle fut détachée de la Hongrie et devint une partie de la Tchécoslovaquie nouvellement créée. C'est donc sous l'autorité tchécoslovaque que la région est organisée en une unité territoriale pourvue d'un nom distinctif, Podkarpatnka Rus, et que des familles hongroises émigrent vers la Hongrie, remplacées par des Tchèques. En 1938, lors la désintégration de la Tchécoslovaquie, la frange majoritairement habitée par des Hongrois se retrouve incluse

1. Les aspects de ce vécu frontalier constituent la trame de la recherche en cours dont ce texte est issu. Elle a débuté en 2008 par quelques semaines, passées à deux reprises dans la ville de Beregovo et dans des villages frontaliers de part et d'autre de la frontière magyaro-ukrainienne. Ce texte s'appuie sur une observation ethnographique, complétée par des dizaines d'entretiens et la lecture de la littérature militante et testimoniale locale, de même que celle des (rares) écrits ethnographiques et sociologiques sur la population transcarpathique. Je remercie mes nombreux interlocuteurs de leur confiance, de m'avoir ouvert leurs maisons, leurs activités et surtout les complexités de leur passé. 
dans la Hongrie, qui annexe quelques mois plus tard le reste de la région. À l'automne 1944, l'URSS force la Tchécoslovaquie à lui céder la région et l'annexe en l'intégrant à la République socialiste d'Ukraine. Lors du rattachement à l'URSS en 1945, son nom sera modifié en Zakarpatska ou Zakarpattia, ce qui signifie « la région au delà des Carpathes » (Magocsi 1978). À partir de cette date, Moscou impose à la Hongrie de se référer à cette région sous le nom de «Karpat-Ukrajna » (Carpatho-Ukraine ) dans toutes les publications, mais le parler commun local et hongrois continue à utiliser le terme Kàrpàtalja, apparu au début du $20^{\mathrm{e}}$ siècle dans les publications savantes. À la faveur de l'effondrement soviétique, celui-ci réapparaît dans l'ensemble des communications écrites, tant parmi les Hongrois de Transcarpathie que par les autorités de Hongrie tandis que le nom officiel en ukrainien reste Zakarpatska.

Les magyarophones habitent pour la plupart les zones agricoles frontalières avec la Hongrie et dans les trois villes importantes de la région, Beregovo (Beregszàsz en hongrois), Munkasevo (Munkacs) et Usgorod (Ungvar). Dans environ 600 bourgs et villages, les magyarophones vivent, parfois entre eux, mais le plus souvent avec d'autres groupes ethniques : les Ukrainiens, majoritaires, les Tziganes, souvent magyarophones, les Ruthènes, prédominant dans les zones montagnardes puis les Slovaques, Roumains et Russes, chacun quelques dizaines de milliers. D'après le recensement de 1989, (Szabo 1993), 78\% de la population régionale est ukrainienne (environ un million) et $12,5 \%$ hongrois. Dans la ville de Beregovo, ces derniers sont majoritaires.

Après la fin de l'URSS, on assiste au réveil et à l'extension des activités des diverses églises, piliers du caractère historiquement multi-religieux de la région. Elles récupèrent alors leurs lieux de cultes et leurs écoles, souvent endommagés, détruits ou recyclés pour des usages industriels et seront rapidement reconstruits. Si l'appartenance religieuse fonctionne ici dans une grande mesure comme marqueur ethnique, très présent dans le parler local, elle peut aussi constituer un lieu inter-ethnique privilégié. Ainsi, la plus grande partie des magyarophones se convertit dès le $16^{\mathrm{e}}$ siècle au protestantisme : d'abord au luthéranisme, puis assez rapidement à la religion réformée calviniste. Toutefois quelques dizaines de milliers, surtout dans les villes, partagent avec les Slovaques l'appartenance au catholicisme, alors que d'autres, tout comme la plupart des Ruthènes et Roumains, disent appartenir à la religion la plus brutalement réprimée de la région et de l'Ukraine par le régime soviétique, en raison d'accusations de " nationalisme » ukrainien et de collaboration avec les Allemands : ils sont uniates (gréco-catholiques). Parmi les pravoslaves (orthodoxes russes), si on trouve aujourd'hui Ruthènes et Ukrainiens, ayant été souvent forcés à la conversion, on ne compte guère de magyarophones.

La présence juive, multiséculaire dans la région, représentait entre les deux guerres une population culturellement et économiquement très dynamique d'environ 86.000 personnes, à majorité urbaine. Et magyarophone. Les survivants de l'Holocauste - à peu près un quart émigrèrent très rapidement aux États-Unis ou en Palestine, plus tard en Israël. Aujourd'hui, les quelques centaines de personnes âgées peuvent, dans les villes de Beregovo, Munkasevo et Ujgorod, pratiquer leur religion dans de petites synagogues urbaines informelles récentes, les anciennes ayant été détruites ou recyclées par les Allemands ou le régime soviétique.

L'identification ethnique de personnes ou familles par la mention de l'appartenance religieuse ou de leur langue principale constitue l'usage majoritaire local en milieu rural, celui qui est considéré comme le plus « poli » : en revanche, le terme " Hongrois » est 
souvent réservé aux occasions de commémorations rituelles ou de discours de type politique : son usage signale le degré de perméabilité du langage local ou personnel aux tournures savantes ou politiques, diffusées par la médiatisation mémorielle magyarophone autour de la déportation au Goulag et des revendications ethniques. En outre, l'idiome des catégories d'appartenance religieuse apparaît comme le seul à pouvoir connoter l'importante population « mixte » : à savoir des personnes nées de mariages inter-ethniques ou ayant des enfants « mixtes ». Cette dimension inter-ethnique de l'organisation sociale locale, déjà ancienne, dont la fonction est aujourd'hui fondamentale dans les activités économiques, garantissant seules la survie, comme le commerce informel, la contrebande et le tourisme, constitue l'un des non-dits importants des discours politiques et mémoriels autour de l'identité « hongroise » de la région.

La multiplicité des toponymies constitue une entrée pertinente pour analyser les contours symboliques de la territorialité du groupe ${ }^{2}$ : en hongrois, la longue absence d'un terme précis reflète l'inexistence d'une identité régionale transcarpathique dans l'espace national hongrois. Mais l'apparition du toponyme Karpatalja, dans les publications savantes et officielles, manifeste la vision d'un centre qui assigne une position marginale à cet espace. Ce terme (« début des Carpates » en hongrois) ne fait sens qu'à partir du point de vue d'un Centre situé à l'Ouest. De même, le terme de Zakarpatska (« au-delà des Carpathes »), reflète la place assignée à la région par une autorité située à l'Est, séparée de la Transcarpathie par les Carpathes. Ainsi, la sémantique de ces dénominations s'inscrit dans la même logique, tout en signalant un conflit symbolique important : les toponymes, hongrois et russe/ukrainien renvoient implicitement à la concurrence entre deux Centres distincts qui imposent des rapports de domination et d'inclusion différents. Le dénominateur commun entre les deux reste la place qu'ils attribuent à la région : celle d'une périphérie.

Cette position écartelée entre Centres concurrents en est venue à marquer autant les représentations territoriales locales que celle du temps social. Bien que depuis plus de 60 ans toutes les toponymies de la région soient officiellement écrites et enseignées en russe, puis en ukrainien, dans le parler quotidien, les magyarophones et la plupart des Ruthènes n'évoquent jamais les lieux et les itinéraires que par leur nom hongrois, tout en utilisant avec aisance les dénominations russes, puis ukrainiennes dans tous leurs contacts avec les institutions. De même, si le territoire de l'Ukraine est placé sous le régime d'un décalage d'une heure par rapport à la Hongrie (et à l'Europe occidentale), les magyarophones et de nombreux Ruthènes et Tziganes de la région règlent cependant leurs montres et horloges, fixent leurs rendez-vous, ouvrent et ferment magasins et échoppes selon "l'heure hongroise ", désignée dans le parler inter-ethnique local, à base lexical russo-ukrainien comme «po misnamu » (heure locale). En revanche, toutes les institutions officielles, entreprises d'État, transports et horloges publiques fonctionnent à «l'heure de Kiev» qui rythme également la vie quotidienne des Russes et Ukrainiens établis dans la région depuis 1946. Si les deux codes temporels sont connus et utilisés ponctuellement par tous, cette dualité du régime temporel trace à coup sûr une frontière identitaire, dessinant deux figures d'allégeance supra-locale.

2. L'analyse qui suit doit beaucoup à l'excellent mémoire de master de David G. Karas (2008) ainsi qu'à nos discussions sur le terrain et à Budapest. Qu'il en soit ici remercié. 
La perspective d'un rattachement politique à l'État hongrois disparut après 1944 ; cependant les magyarophones de Transcarpathie ont continué à se représenter comme partie d'une unité nationale hongroise qui transcende les frontières internationales imposées après 1918. Cette représentation fut puissamment étayée par la déportation massive dans les camps du Goulag dont ils devinrent les cibles en tant que citoyens hongrois, dès l'occupation, puis l'annexion soviétique de leur région : elle a renforcé une représentation victimaire et résistante de cette appartenance. Par la suite, pour la reconstruction des identifications à l'échelle locale, la catégorie de « hongrois » restait également légitime pour les populations car elle permit une sauvegarde et une certaine reproduction des structures sociales des traditions de socialisation, du capital culturel et linguistique et des solidarités de la communauté, dans le domaine privé, tandis que les catégories et modèles imposées par le haut dans le domaine public ou politique ont été discréditées par les vagues de répression. Ce processus confirme l'analyse développée par Rogers Brubaker (2007), à propos de la recomposition ethnique en Transylvanie, sur la coexistence de plusieurs domaines, parfois discontinus et contradictoires de l'ethnicité : le domaine du pouvoir, celui d'une ethnicité politique, et le domaine de la solidarité, celui d'une ethnicité « du quotidien ».

Ce sont ces tiraillements entre relations d'allégeance économique, politique et culturelle concurrentes qui semblent dessiner les contours d'une singularité régionale transcarpathique qui englobe tous les groupes ethniques. Devenue à l'issue de son détachement de la Hongrie une périphérie, tributaire de plusieurs Centres, toujours éloignés, c'est la permanence de cette position de dépendance périphérique qui finit par produire une territorialité particulière, marquée par la fragmentation des allégeances face à plusieurs sphères d'influence avec lesquels elle partage des passés différents et entretient des rapports changeants.

\section{De la minorité nationale à l'ethnicité périphérique}

Si les politiques aussi brutales que contradictoires de l'URSS ne sont jamais parvenues à anéantir la centralité culturelle de l'appartenance supra-locale magyare, ce sont paradoxalement les recompositions géopolitiques d'après 1989 qui semblent mettre à mal ce lien culturel d'identification à la Hongrie, fondement d'un profil de minorité nationale. À la faveur d'une relation que Budapest souhaite particulièrement cordiale avec Kiev, un Traité bilatéral est signé en 1991 où la Hongrie s'engage à abandonner toute prétention territoriale et confie le sort des Hongrois de Transcarpathie à la bienveillance de l'État ukrainien. Ce traité est vécu comme une véritable trahison parmi les élites hongroises locales et dessine pour les Hongrois de Transcarpathie l'image nouvelle et conflictuelle d'une Hongrie en tant que pays étranger.

Enfin l'intégration de la Hongrie dans l'UE et dans l'espace Schengen, qui impose des formalités administratives très lourdes aux citoyens ukrainiens - y compris donc les Hongrois ethniques - voulant se rendre, s'installer ou étudier en Hongrie semble avoir diffusé dans l'ensemble de la population une désillusion, qui s'est progressivement muée en méfiance envers l'État hongrois.

Aujourd'hui, sans abandonner leur identitification comme Hongrois, les magyarophones de Transcarpathie ont intégré le fait qu'ils ne faisaient pas partie de la même communauté politique que les Hongrois de Hongrie. En revanche, depuis la fin de l'URSS, l'intense activité commerciale informelle et une contrebande multiforme - ressources économiques fondamentales pour la survie dans la région - ont densifié et diversifié les interactions inter- 
ethniques entre Ruthènes, Ukrainiens et magyarophones locaux, tout en activant et élargissant les réseaux familiaux et d'affinité de part et d'autre de la frontière hungaro-ukrainienne : ces pratiques ont étendu et prolongé l'espace social pluri-ethnique de la Transcarpathie vers les départements frontaliers du Nord-Est hongrois par l'installation de familles et de divers commerces transfrontaliers, transformant le paysage urbain et social de ses bourgs et villes. Ces pratiques multiformes, qu'aucune disposition policière ou réglementaire ne parvient à stopper, dessinent les contours d'une identité régionale transfrontalière, où l'échelle de prestige se déplace vers les compétences sociales de négociation, de mobilisation de multiples ressources culturelles, identitaires et d'interaction. Parallèlement en Transcarpathie, discours et pratiques (notamment festives, de voyage, et de création et d'utilisation d'espaces virtuels) des générations plus jeunes témoignent d'une identification progressive envers le territoire transcarpathique lui-même, comme référent d'origine, qui marque l'émergence d'une identité magyarophone de Transcarpathie.

Dans ce processus, la Transcarpathie, au lieu d'être perçue comme un territoire artificiellement séparé d'un territoire hongrois commun - celui d'avant 1918, et celui d'aujourd'hui - devient le référent principal dans le passage d'une logique de minorité nationale à celle d'une ethnicité locale qui rend disponibles de nouveaux ancrages identitaires : le territoire régional de Transcarpathie «par le bas », comme terreau d'une ethnicité hongroise locale, et « par le haut », la figure de la « nation hongroise » qui désigne dans les gloses locales une communauté méta-territoriale de l'ethnos disséminé dans plusieurs États, dont le territoire immatériel est la langue hongroise.

Ainsi, l'assouplissement post-soviétique des frontières d'État, en faisant voler en éclats l'idéal d'un homeland englobant, soigneusement conservé et transmis par la mémoire familiale pendant la période soviétique, finit par faire émerger à la place de la frontière d'État hermétique du passé, de nouvelles frontières culturelles entre forme étatique et formes ethniques de la " magyarité ». Cependant, la tension entre ces deux types d'identification - vis-à-vis de l'ethnicité hongroise (et de l'État hongrois) d'une part, et du territoire transcarpathique d'autre part - est permanente et se manifeste à plusieurs niveaux.

Les fêtes commémoratives sont des moments privilégiés de la mise en scène de cette tension. Ainsi, le 15 mars, qui commémore la révolution hongroise de 1848 contre les Habsbourg, continue à être considéré comme la fête la plus importante pour les Hongrois de Transcarpathie, dont la célébration implique souvent la présence de politiciens hongrois invités. D'une part, elle sacralise un moment historique d'unité entre le territoire de la Hongrie d'avant 1918 et la nation ethnique hongroise, commémorée pour sa lutte pour l'indépendance nationale. Dès lors, la célébration locale de cette fête continue à ancrer la mémoire historique des magyarophones de Transcarpathie dans une mémoire partagée, rappelant une unité perdue. D'autre part, cette fête est perçue dans l'ambivalence, et non seulement par les Ukrainiens : car c'est aussi la fête nationale de la Hongrie, ce qui rend l'enjeu de la commémoration équivoque. Cependant, la présence d'un drapeau tricolore hongrois, dépourvu de la croix de Lorraine, considérée comme blason de l'État, est glosée dans les discours des dirigeants politiques locaux, et perçue par la majorité, comme le symbole de la communauté virtuelle de tous les Hongrois, disséminés par le monde, celui de la " nation », débordant et transcendant les frontières de l'État hongrois. Ainsi, l'interprétation, interne ou externe aux groupes magyarophones, peut osciller entre celle d'une mise en scène de l'unité des Hongrois ethniques par-delà les frontières, ou celle d'une affirmation identitaire de portée 
locale qui permettrait aux magyarophones de Transcarpathie de redessiner symboliquement les frontières de la « communauté » vis-à-vis des autres groupes.

Dès lors, l'ethnicité hongroise de Transcarpathie se révèle être une construction complexe : peu à peu, la Transcarpathie elle-même devient un homeland, le territoire identitaire privilégié d'une ethnicité hongroise locale en voie de construction. Parallèlement, les fêtes commémoratives des magyarophones de Transcarpathie évoquent et mettent en scène de plus en plus souvent une appartenance supra-locale à une communauté déterritorialisée, englobant tout l'ethnos hongrois, alors que l'impossibilité d'une solidarité forte avec un motherland, terre des origines, État aux stratégies totalement différentes de leurs buts constitue un leitmotiv des discours quotidiens. Si la dimension maternelle est récurrente dans les termes hongrois couramment utilisés d'Anyaorszag ("pays-mère ») mais aussi de Szülöföld (littéralement « la terre qui donne naissance », la terre de naissance), le premier, désignant la Hongrie, est progressivement mise à distance dans les identifications locales. Ainsi la Hongrie comme Anyaorszàg, ne recouvre, ni ne contient plus symboliquement la région transcarpayhique représentée désormais comme le szülöföld (terre de naissance). Cette dernière connote en hongrois, la part affective du vécu du haza (patrie), appartenant à la fois à l'intimité individuelle privée et à celle, culturelle, partagée. Mais l'assimilation de la Transcarpathie à cette représentation est récente et montre que le territoire local, comme ancrage à la fois de référence et d'appartenance remplace progressivement celui de la Hongrie.

Ainsi, la position périphérique de dépendances multiples modèle les contours de la territorialité transcarpathique jusqu'à ce qu'il devienne lui-même le territoire privilégié de l'identification ethnique. Mais aujourd'hui cette périphérie apparaît, du point de vue des acteurs locaux, comme étant «sans Centre », car l'adhésion magyarophone à des collectifs politiques ou nationaux plus larges est devenue mitigée. Aucun n'est perçu comme entièrement légitime ou capable d'assumer ce rôle, ni l'ensemble des Hongrois ethniques qui constitue une espèce de « communauté culturelle imaginée » sans espace politique commun, ni l'État hongrois dont les priorités sont étroitement liées à son intégration européenne, ni l'État ukrainien perçu comme étranger et pour lequel l'infime minorité hongroise ne constitue pas une question primordiale. Ainsi, la Transcarpathie devient elle-même productrice d'une ethnicité hongroise locale. Cependant celle-ci est ancrée dans un territoire périphérique dominé que les multiples pratiques commerciales informelles et illégales étendent au-delà de la double frontière - celle entre deux États-nations et celle de l'Union Européenne - et en font une région multi-ethnique transfrontalière.

\section{Itinéraires d'une élite : de la mémoire à la visibilité}

Après le début d'une déportation massive qui a pour longtemps scellé les rapports entre la communauté hongroise et leur nouvel État, une série de nouvelles mesures politiques ont contribué à ébranler la société locale : l'épuration, la dékoulakisation, la collectivisation des terres et des moyens de production, l'interdiction de l'Église gréco-catholique fondue dans l'Église orthodoxe et la transformation du système éducatif. Étant donné que les Hongrois de Transcarpathie n'étaient pas géographiquement isolés des autres « nationalités »- ce qui excluait une administration spécifique - et qu'ils ne disposaient pas non plus de leurs propres institutions politiques alors même qu'ils constituaient une minorité importante et, 
dans beaucoup de localités, une majorité ethnique, le pouvoir soviétique disposait avec la politique d'éducation d'un levier souple et efficace ${ }^{3}$.

Si les écoles primaires hongroises n'ont pas été fermées et ont assuré l'éducation sans interruption, les autres niveaux d'enseignement ont été rapidement étatisés et refondés en russe avec des programmes soviétiques. La politique éducative a donc constitué pour les autorités soviétiques un domaine privilégié de contrôle et de normalisation de la minorité hongroise, tandis que pour les Hongrois, la lutte incessante pour une éducation dans leur langue devint la caution de la continuité de la reproduction culturelle.

Si auparavant il s'agissait de briser un groupe ethnique, perçu comme inconciliable avec le projet soviétique, après la mort de Staline, la politique soviétique semble chercher davantage à fabriquer une communauté hongroise soviétique. Mais cette politique, loin de désorganiser les identifications magyarophones, permit au groupe d'investir des institutions - l'éducation en général et la nouvelle faculté magyarophone de philologie en particulier, preuve de la nouvelle orientation de la politique soviétique. Cette faculté donna naissance à une élite intellectuelle magyarophone qui émergea après les années 1960 : elle offrit de fait un espace commun à l'auto-organisation du réseau de celle-ci. En cherchant à particulariser la communauté hongroise de Transcarpathie par rapport aux Hongrois de Hongrie à travers son éducation, son orthographe, ses patronymes ${ }^{4}$, à constituer en somme une culture soviétique de langue hongroise, les autorités soviétiques ont en fait renforcé la solidarité ethnique de la très minoritaire élite hongroise qu'ils pensaient assimiler.

Rapidement, les intérêts de ces universitaires débordent le strict cadre littéraire vers des activités de type ethnographique parmi lesquelles des recueils de contes populaires, de poésie, danses et chansons folkloriques, parfois assorties de tentatives d'analyse sociologique. Ces retrouvailles avec un milieu rural dont la plupart sont originaires, médiatisées par l'écrit et par leur nouveau statut d'intellectuels, ont fait émerger, filtrées par le biais folklorique, des bribes de récits et de réminiscences de la déportation massive au Goulag, ressentie à la fois comme fondement de la cohésion interne du collectif hongrois et comme l'événement fondateur de son inclusion forcée dans l'espace soviétique. Ces universitaires privilégiés - car bénéficiant d'une mobilité sociale inaccessible à leurs aînés - redéfinissent alors progressivement leur propre identité, en la projetant autant qu'en la puisant dans le terreau des traditions locales. Leur identification aux paysans qu'ils interrogent offrit à ces jeunes intellectuels un ancrage dans une communauté plus large, pouvant servir de système de contre-légitimation vis-à-vis du régime soviétique.

Si la politique éducative plus libérale après 1953 a échoué à produire une élite hongroise entièrement loyale au régime, il serait faux d'en conclure que l'identité ethnique hongroise n'a pas été fondamentalement reconfigurée dans le contexte soviétique, notamment par cette nouvelle élite intellectuelle qui est devenue, dès la fin des années 1970, l'acteur principal de

3. Le cas des Hongrois n'est pas particulier : toutes les minorités soviétiques ont été administrées ainsi en Transcarpathie comme ailleurs. Le cas des Hongrois en Transcarpathie est similaire à celui des Ruthènes, des Roumains, des Slovaques, etc. Même si les Hongrois de Transcarpathie vivent dans une région facilement identifiable, présents dans pratiquement toutes les villes et les villages de la Transcarpathie, pour les autorités soviétiques ils constituent la composante ethnique la plus « sensible » du fait de l'attachement historique de la région à la Hongrie. Dès lors, ils deviennent les cibles privilégiées des politiques éducatives nouvelles qui visaient à mater les résistances potentielles.

4. On a aussi introduit l'usage du second patronyme, usité en russe mais inconnu en hongrois. 
la construction d'une mobilisation : elle en a défini les discours, les stratégies et les cadres institutionnels (Karas 2008 : 75). Cependant, si elle a pu en devenir la force motrice, c'est précisément en raison de son intégration dans le système institutionnel soviétique.

Mais le passage progressif du club informel de discussions littéraires à celui de groupe de collecte ethnographique, et enfin à celui de groupe politique, a été conditionné aussi par la pression grandissante que les autorités déployèrent pour reprendre le contrôle d'un espace qui tentait de s'autonomiser. Loin d'être une simple machination des élites luttant pour étendre leur pouvoir, le succès de la mobilisation, parti d'une dizaine d'étudiants dans les années 1960 et rassemblant pratiquement toute la population hongroise active en 1989$1990^{5}$ peut s'éclairer par l'attrait d'un espace ethnique, susceptible de rétablir ce qui est perçu comme une continuité historique rompue par la contrainte : l'appartenance référentielle à la « nation » hongroise, sinon à l’État.

Dans cette expansion réticulaire d'une mobilisation, comme dans d'autres contextes nationaux, le combat pour et par la mémoire a joué un rôle pivot de médiateur et de lieu de rencontre entre l'élite et les groupes locaux, grâce aux réseaux familiaux, d'interconnaissance et à celui des « clubs culturels » locaux. Leur mise en récit écrit et diffusion par les réseaux d'intellectuels opère dans ce contexte social le reclassement progressif des souffrances individuelles et familiales dues au communisme, en autant d'emblèmes de la blessure d'une communauté, punie pour son appartenance à une nation d'origine. Devenue ainsi pièce maîtresse d'une mémoire collective magyare en construction, à l'effacement totalitaire antérieur des traces et de la mémoire des personnes déportées, a succédé l'effacement posttotalitaire des profils individuels derrière la communauté nationale victime.

En effet, le déferlement ininterrompu dès 1991 de discours rituels commémoratifs médiatisés et de recueils de récits de déportation, souvent signés par des figures intellectuelles dirigeantes du mouvement, suivis d'érudits locaux, a fixé progressivement les conventions narratives et interprétatives d'un « bloc mémoriel » autour d'une expérience " hongroise » du Goulag, articulé sur des figures rhétoriques stéréotypées dont la répétition les ritualise. Ainsi la phrase attribuée à Staline « La question hongroise est une question de wagons » ou une phrase issue du roman d'un écrivain local, massivement et souvent clandestinement lu dans les années soviétiques : « leur seul crime était d'être Hongrois. » Il s'agit d'une figure mémorielle unifiée, de victimisation ethnique collective, figure construite par la sélection et la canonisation d'évènements et de comportements emblématiques, susceptibles de servir de légitimation, faire-valoir et base de revendication collective. Cette production visait autant à obtenir l'aval et l'appui de certaines composantes de la sphère politique hongroise qu'à négocier des concessions politiques avec l'État ukrainien.

Les premières transcriptions des récits locaux, sauvegardant minutieusement le style narratif tissé d'allusions, de tournures populaires parfois humoristiques et d'émotions rentrées (par exemple Punyko 1993), faisaient encore apparaître conflits et soupçons internes dans les villages, entre magyarophones locaux, sympathisants pro-soviétiques, communistes et anticommunistes, complicités et solidarités inter-ethniques et inter-religieuses dans l'épreuve du Goulag, de même que la découverte chargée d'émotions contradictoires de la profonde misère et parfois de la solidarité des Russes libres, autour des camps. Elles furent rapidement

5. Le KMKSZ compte alors plus de 40.000 membres, voir Tiz év a magyarsag szolgalataban, KMKSZ X. kôzgyûlése, Ungvar : 1999. 
suivies par des récits recomposés dans un style uni savant, et épurés autour du fil rouge d'un martyre ethnique d'un collectif hongrois entièrement soudé et uni, sans aucune division interne, ni contacts ou complicité avec d'autres groupes (Balog 1992 ; Bottlik 1997 ; Dupka 1993a, 1993b ; Nagy 1992 ; Szebeni 1991).

Entre-temps, le consensus postcommuniste a progressivement laissé place à une atomisation de la communauté de mobilisation : peu à peu, la mémoire publique ainsi construite devint la seule passerelle et point de convergence dans un processus d'antagonisation des divergences politiques. Ce processus culmina par l'implosion du mouvement unitaire qu'a été le KMKSZ des années 1989-1990 : c'est dans la formulation des choix stratégiques, notamment des modes de partenariat et de dépendance vis-à-vis de diverses forces politiques hongroises et ukrainiennes que les différences se creusèrent. Ces choix différenciés créèrent des identités politiques multiples que le but commun, la défense des droits collectifs, ne parvint plus à rassembler : l'ethnicité politique hongroise se polarisa.

En 1996, les groupes qui se sont progressivement retirés du KMKSZ décident de fusionner pour former une alternative à ce dernier qui dispose toujours de la meilleure structure avec une implantation à tous les échelons territoriaux, vestiges des anciens clubs culturels qui se sont mués en sections locales et régionales. Dès lors, avec ces scissions, l'unité du monde social magyarophone s'émietta en sociabilités politiques, organisés comme des réseaux rivaux. La logique de la compétition politique entre deux partis, tous deux dépendants d'alliés nationaux hongrois et ukrainiens, brise souvent les anciennes solidarités et va élargissant son emprise sur l'ensemble de l'espace social, jusqu'au niveau local. Ainsi, chaque camp devient un super-réseau quasi autarcique et corporatiste, ayant des organisations spécifiques pour les enseignants, écrivains, journalistes, avocats (Karas 2008 : 84). Les acteurs se plient à cette contrainte, pour avoir accès à des ressources financières et symboliques de légitimation, extérieures à la région et en quantité limitée : notamment les subventions qui viennent de Hongrie.

Aujourd'hui, les élites influentes dans chaque camp sont celles qui ont réussi à cumuler différents rôles et statuts, à jouer sur plusieurs plans, à conserver des attaches dans plusieurs activités et domaines professionnels et politiques : aussi est-il difficile d'opérer ici la distinction classique entre élite politique, économique, administrative et intellectuelle.

Dans ce contexte de clivage d'un monde social dont ces partis-réseaux prétendent encore tous à assumer la sauvegarde et la reproduction, le seul bien symbolique commun des sociabilités politiques rivales reste la mémoire publique " hongroise » centrée sur la déportation, de plus en plus ritualisée. Si cette dernière a fini par influencer en retour les modes locaux, de remémoration, ceux-ci subsistent et se reproduisent encore, alors que des initiatives touristiques locales s'attachent à créer de nouveaux « lieux de mémoire » et exploitent ceux, déjà créés, comme ressources patrimoniales.

\section{Les années de la peur : souvenir et oblitération autour du " malenki robot »}

L'oblitération, loin de se confondre avec l'oubli, effacement d'un élément de la mémoire, constitue une mise en latence collective du souvenir d'un événement, en en sauvegardant des marqueurs indirects, est un processus culturel socialement construit, qui apparaît comme une modalité particulière de la mémoire collective. Elle se distingue également du secret, car ce qui est éludé ou évoqué allusivement, fait partie au contraire d'un savoir commun localement partagé et non explicitement interdit d'évocation à l'intérieur du groupe. Ainsi, dès 1945, 
sous le poids d'une répression brutale, les collectifs magyarophones de Transcarpathie, à l'instar d'autres populations de l'URSS, ont dû recouvrir par la chape d'un silence, imposé de l'extérieur, l'évocation explicite du déroulement des arrestations, des étapes pénibles et du travail forcé de la déportation, du déclassement, de la misère ou des harcèlements des familles, de la mort lointaine et souvent non communiquée des déportés, puis des modalités de retour et réinsertion des survivants. Dès cette époque, le terme local usuel condensant et évoquant l'ensemble de cette expérience collective sera celui, issu du russe, de malenki robot. Signifiant dans un russe mal perçu littéralement «petit travail », l'étymologie populaire l'attribue aux soldats et autorités soviétiques ramassant les civils sous le faux prétexte d'un court travail de déblaiement de deux trois jours : cette expression associe ainsi cruauté et tromperie au collectif russe.

Si le respect forcé des codes soviétiques du non-dit de la répression constitua l'expérience commune de toutes les composantes de la société soviétique, les cachettes, au sein de l'intimité familiale, contenant de témoignages matériels des déportés - objets personnels, papiers et photos antérieurs, cartes envoyées des camps - semblent avoir constitué autant des reliques familiales, que des ancrages d'une silencieuse intimité culturelle au sein du collectif hongrois, à l'instar d'autres groupes victimes. Ces reliques furent ensuite enrichies par des objets usuels des camps, des poèmes, chants, prières, listes de compagnons décédés et notes que certains survivants mirent en écriture après leur retour.

Aujourd'hui, le mode de visibilité de ces objets testimoniaux s'est diversifié et ritualisé. D'une part, ils occupent, le plus souvent, un lieu particulier, entre intime et public, dans la maison : si une photo du disparu est souvent exposé dans la pièce commune, ornée d'une fleur ou d'une bougie, les documents écrits, et objets usuels soigneusement regroupés et séparés d'autres, se trouvent dans les chambres à coucher, prêts à être ressortis pour les visiteurs. Lors des commémorations annuelles, à l'église, au cimetière ou devant le monument local, les souvenants âgés se présentent souvent, portant des photos, notes ou objets usuels du Goulag, ayant appartenu au disparu. S'il n'existe aucun musée, consacré à la déportation dans la région, un certain nombre de ces objets furent donnés au petit musée historique de Beregovo, construit sur des collections hétérogènes d'un intellectuel local magyarophone.

En raison de la répression soviétique, l'état, les caractéristiques et les changements du savoir narratif portant sur le Goulag, latent pendant les quatre décennies avant la perestroïka, resteront à jamais dans l'ombre. Cependant, les premiers recueils de récits des années 1980, réalisés par des jeunes intellectuels locaux, de même que les premiers travaux d'ethnographes hongrois (par exemple, Fejös 1995), font état de conventions narratives suffisamment élaborées dans l'évocation du lager et du malenki robot pour signaler une tradition orale familiale vivante, dont le style de narration rappelle celui, local, des conflits quotidiens et celui des récits de soldats hongrois de la Première Guerre mondiale (Fejös 1995).

Par ailleurs, un certain nombre de poèmes, prières et ballades en hongrois, nés dans les camps du Goulag, s'intégrèrent dans la tradition orale locale, se cachant souvent parmi les chants religieux ou folkloriques. La « Prière des mille prisonniers » en six strophes - recueillie dans nombre de villages et de bourgs - fut créée collectivement à Noël 1944 dans le camp de rassemblement de Szolyva en Transcarpathie ; le poème d'un lycéen, intitulé « Noël de prisonniers ", écrit également à Szolyva où les épouses et mères, ayant pu visiter une fois leurs proches avant le départ en Sibérie les ont appris par cœur, diffusé dans les familles puis chanté sur les mélodies de psaumes dans l'église. Un chant funéraire de l'Église réformée, 
écrit dans le village de Szaloka par des survivants revenus, en commémoration des déportés morts du village s'est également diffusé, chanté sur la mélodie du psaume 35 . Il semble que des poèmes chantés, localement appelés «ballades de lager » ont pu se mouler dans les chants et ballades populaires traditionnels (Punyko 1993 : 12). Diffusés et folklorisés, ou constituant un patrimoine mémoriel, passé directement de l'intimité familiale à la publication militante et/ou savante, ces textes signalent, sous l'épaisse couche de " secret » officiel, un processus continu d'appropriation familiale et collective de la continuité mémorielle.

En général, les familles finirent par apprendre la mort du déporté, sans en connaître le lieu, ni la date exacte. L'absence définitive du cadavre et l'impossibilité de l'enterrement furent vécues comme une amputation du patrimoine mémoriel familial et villageois, engendrant des pratiques ritualisées de substitution. Si le chant réitéré du psaume funéraire de Szaloka dans l'église locale peut être interprété comme une sorte d'enterrement déterritorialisé, ériger un poteau funéraire - traditionnellement en bois au sein de la population magyarophone réformée de la région - à un défunt déporté n'était pas possible sans danger. D'aucuns se souviennent cependant de noms de morts gravés sur le poteau familial, mentionnant parfois « décédé au loin » ou " décédé en lieu inconnu », mais les épitaphes, lisibles aujourd'hui dans les cimetières de villages et de bourgs, furent créées dès 1987 dans le style poétique traditionnel par l'écrivain local d'épitaphes. Ailleurs, on a placé une plaque, commémorant tous les déportés du village (Gôdényhàza), mais le secrétaire du parti a ordonné son enlèvement.

Aujourd'hui, dans le discours des «souvenants » et de leurs descendants, ces chants, poèmes et prières mais aussi les nombreux récits et notes d'époque recueillis et publiés, les monuments publics et les commémorations annuelles apparaissent tous, à la fois comme créateurs de tombes immatérielles, restituant la trace des absents, comme justice rendue aux victimes innocentes et comme réparation d'une déchirure dans le tissu généalogique du groupe. Mais la lecture de ces livres, leur médiatisation dans les médias locaux et hongrois et l'enseignement scolaire local influencent en retour certains cadres expressifs et interprétatifs de la mémoire locale. Ainsi, le déficit de territorialisation que signale l'absence d'enterrement et l'impossibilité persistante de connaître avec certitude la date et le lieu des décès s'interprète de plus en plus couramment comme signe d'une mort héroïsée, d'un destin moralement exemplaire. Pour les plus jeunes, sans souvenir, la mémoire, de familiale se transforme souvent désormais en livresque et rituelle : elle semble donc redessiner à l'infini la figure d'un martyre hongrois collectif sans visage, exprimé dans un registre victimaire d'inspiration chrétienne.

Mais si dans l'évocation informelle et orale du passé, coexistent des composantes mémorielles multiples teintées par l'ambivalence, télescopant temporalités, espaces et registres divers, leur mode d'énonciation épouse parfois les nouvelles normes mémorielles écrites. En comparaison avec les récits recueillis au début des années 1990, actuellement, dans les générations adulte et âgée, des bribes de récits sur des événements sanglants de la guerre, avant l'occupation soviétique, sur ceux, ultérieurs, de la collectivisation et la dékoulakisation, suivis de graves pénuries, sur le quotidien de la répression, semblent de plus en plus « aspirées » par le bloc mémoriel de la déportation et sa trame narrative : elles émergent rarement sans y être insérées et elles y apparaissent dépourvues de repères chronologiques ou spatiaux propres. Si la chronologie et les changements de la période soviétique sont ainsi de plus en plus neutralisés et « noyés » dans l'expérience emblématique du Goulag, l'évocation 
du partage avec d'autres groupes de ces épreuves ultérieures devient aussi plus ténue et difficile et son potentiel de solidarité et d'identification s'affaiblit.

Dans les allusions très codées sur l'existence et l'identité de dénonciateurs et complices de la répression soviétique locale - ayant été souvent déportés eux-mêmes - ils continuent à être évoqués dans l'idiome traditionnel de référence à des acteurs ambivalents du passé : jamais nommés, mais spécifiés soit par le village d'origine, soit par la langue maternelle, soit par l'appartenance religieuse. Si ces allusions, disparues des récits plus récemment publiés, apparaissent encore dans les différents entretiens comme renvoyant à une trame latente d'accusations mutuelles, paradoxalement leur caractère à peine esquissé et jamais public perdure jusqu'à aujourd'hui. Ainsi, le silence imposé par le régime soviétique semble s'être transformé en une oblitération interne, partagée entre élites savantes, produisant la mémoire écrite, et locuteurs locaux : son double but paradoxal semble être à la fois de préserver localement des rapports interfamiliaux et inter-ethniques d'échange et de collaboration économique, indispensables pour la reproduction du groupe et de conforter une unité ethnique post-soviétique fondée sur la victimisation.

Point de convergence entre mémoire informelle et mémoire publique et écrite de l'année 1944, l'épais silence, sans marqueur mémoriel indirect, qui recouvre le plus souvent la disparition de l'une des composantes, dynamique et majoritairement magyarophone de la population régionale : bourgeois, intellectuels, commerçants et même agriculteurs, les Juifs transcarpathiques urbains et ruraux. Leur dépossession et déportation massive dans les camps de concentration nazis, œuvre conjointe des Allemands et de la gendarmerie envoyée du centre de la Hongrie, a précédé de peu le début de l'occupation et de l'annexion soviétiques. Questions ou allusions se heurtent presque toujours à un déni de mémoire et de savoir («je ne me souviens pas », « je n'en ai pas connu », « je ne le savais pas »). Malgré le témoignage des archives et celui de traces architecturales, désormais à peine visibles de la présence culturelle juive, l'association de ce qui est souvent nostalgiquement nommé " l'époque hongroise » à des massacres et exactions semble dessiner pour les souvenants la figure insupportable d'un bourreau nazi, en partie hongrois. Elle serait susceptible de fissurer douloureusement l'image de l'ancien motherland, la Hongrie, de même que l'unité ethnique et identitaire créée autour d'un récit fondateur victimaire dans la mobilisation mémorielle collective de la fin de l'URSS.

Ce silence dessine les limites des paradoxes que peut porter une mémoire sociale (Collard 1989), dont la composante informelle constitue une sorte de mosaïque mémorielle, dotée d'une chronologie mouvante qui peut condenser événements et acteurs divers dans l'intemporel d'une seule expérience dramatique emblématisée. Elle est faite d'un savoir implicite - diffusé par les souvenants et par des objets ou reliques mémoriels - engendrant et alimentant un mode d'énonciation particulier, soutenu par des locutions et tournures spécifiques, condensatrices de l'évocation, et de modes ritualisés de conduite et d'actions symboliques. Cette figure mémorielle se produit le plus souvent en contrepoint et parfois en contestation d'idéologies et d'historicités dominantes et officielles, ce qui augmente encore sa charge émotionnelle. L'attribution de ces pratiques et représentations idéologiques hostiles, comme propre non pas à un système transnational mais à un persécuteur collectif national ou ethnique, ethnicise en retour l'identité des victimes. 


\section{La symbolique des monuments commémoratifs}

Le symbolisme des plaques et monuments commémoratifs, érigés pour les cérémonies de 1989 et par la suite éclaire le double rapport de la construction mémorielle publique avec l'espace culturel et politique local et avec celui, national, hongrois.

Le combat identitaire et politique par symboles et représentations interposés pour le marquage et la «possession » symbolique des espaces centraux de villes et villages est amplement documenté (Aguilhon 1978), notamment dans les études portant sur les sociétés post-communistes ou en crise de légitimité politique (Hofer 1992). En 1989-90, les autorités locales soviétiques de la Transcarpathie ont très souvent empêché, autant la célébration de la cérémonie que l'érection d'un monument commémoratif dans les centres-villes ou sur la place principale des villages. Ainsi, de nombreuses cérémonies et monuments datant de ces années ont investi les églises et les cimetières. Si en raison du marquage ethnique local de l'appartenance religieuse, ces derniers continuent à constituer les espaces sociaux et symboliques privilégiés de l'intimité culturelle (Herzfeld 1997), le caractère funéraire de la commémoration y ajouta une légitimité supplémentaire, perpétuée par des plaques ou pierres tombales commémoratives, portant les noms des disparus et objets de commémorations annuelles suivies d'un office religieux.

À partir de la fin de l'URSS et de la naissance de l'État ukrainien, le marquage symbolique de lieux emblématiques de la « magyarité » investit progressivement une multiplicité d'espaces ruraux et urbains. L'érection de monuments commémoratifs non figuratifs ou de statues semble suivre les normes esthétiques des monuments européens aux victimes des guerres mondiales (Agulhon 1978), en activant parfois des motifs issus de la tradition régionale, comme le $f e j f a$, bâton funéraire sculpté par des artisans locaux, élément traditionnel de la culture locale issu du calvinisme populaire. Ce symbole funéraire a connu au $20^{\mathrm{e}}$ siècle une extension urbaine et un élargissement de sens : autant en Hongrie qu'en milieu minoritaire ou émigré, il en est venu à commémorer victimes et héros d'événements tragiques de l'histoire nationale (Hofer 1992). Exemplaires de ce processus, des fejfa érigés ici en mémoire des déportés semblent inscrire dans le paysage et la mémoire régionaux la représentation supraétatique d'une « magyarité » sans frontière dont l'un des motifs, remontant au $19^{\mathrm{e}}$ siècle, est un destin national tragique partagé.

Les monuments les plus impressionnants, investissant des lieux centraux ou auparavant non marqués, sont le « Parc du Souvenir » érigé à Szolyva, à la place du camp de transit des déportés transcarpathiques - solennellement inauguré en 1994, cinquantenaire de la déportation - et le monument du village de Tiszapéterfalva, réalisé en 1990.

$\mathrm{Si}$, surtout au début des années 1990, plaques, pierres tombales et monuments furent réalisés par l'initiative et les donations conjointes d'acteurs locaux et d'organisations ethniques, ces dernières, mobilisant politiciens et partis hongrois alliés, vont se réappropriant et sacralisant progressivement des lieux de mémoires régionaux emblématiques d'un passé hongrois plus lointain, comme si le foyer mémoriel construit autour du Goulag, ouvrait la voie à la réorganisation et re-territorialisation de tout un paysage commémoratif, offrant un contexte interprétatif plus large aux événements collectifs plus récents. Ainsi, dès le début du combat politique pour l'introduction de l'enseignement de l'histoire hongroise dans les écoles de la région, furent rénovés et dotés de plaques commémoratives des lieux de mémoire plus anciens, comme ceux liés à l'insurrection anti-Habsbourg dirigé par le prince Ferenc Rakoczi, originaire de la région, ou le château fort de Munkacs (Munkasevo), lieu d'une 
longue résistance contre l'assaut des Turcs, tous ritualisés par des commémorations annuelles. De même, dans l'un des lieux les plus emblématiques de la mythologie nationale hongroise, le col de Verecke dans les Carpathes, réputé avoir été le lieu d'arrivée dans le bassin des Carpathes des tribus dont la sédentarisation, l'alliance et la conversion au christianisme furent les fondements du royaume millénaire fondé par Saint-Etienne, fut récemment reconstruit un monument commémoratif massif. Son existence intensifia encore ce que l'on peut appeler un «tourisme patriotique », pèlerinage laïque mais ritualisé d'individus, de familles et de groupes, de Hongrie et de magyarophones d'autres pays, se recueillant sur des lieux, considérés comme ancrages identitaires ethniques débordant les frontières étatiques.

Ce marquage et cette ritualisation d'espaces interpatriotiques (Losonczy 1997), parce que d'autres groupes ethniques, comme les Ruthènes, ou les Ukrainiens « nationaux » les investissent également comme les leurs, continuent à alimenter de " contre-rituels » politiques comme l'endommagement, le déboulonnement des monuments ou la construction de marquages rivaux, accompagnés de polémiques dans les médias et parfois même d'incidents diplomatiques. Cette figure de l'histoire territorialisée, transformée en expérience mémorielle par des visites touristiques et des rituels commémoratifs, parfois rivaux, semble être l'élargissement d'une mémoire publique ethnicisée, car elle porte un double enjeu paradoxal : soutenir symboliquement à la fois des reconstructions nationales rivales, ancrées dans des états, et des reconstructions ethniques régionales, s'identifiant à une unité ethnique supra-locale transfrontalière.

\section{En guise de conclusion}

Les rituels politiques de portée ethnique (Kertzer 1988) apparaissent comme des dispositifs privilégiés pour condenser les mémoires personnelles, familiales et sectorielles en une figure unitaire, les transformant ainsi en mémoire publique exemplaire, emblème d'une communauté. Mais en explicitant l'implicite et le flou de la mémoire sociale, la mise en écrit savante, la patrimonialisation d'une doxa mémorielle ethnicisée et surtout sa ritualisation, changent la nature de la mémoire collective : de bribes d'expériences fragmentées, souvent contradictoires, portant une conflictualité latente et traversées d'oblitération, elles la transforment en un savoir explicite collectif incontestable, pièce maîtresse d'une unité ethnique idéale, au-delà de toute division, conflit ou ouverture vers d'autres groupes. Cette transformation savante et ritualisée de la souffrance en victimisation engendre un mode d'énonciation normatif et stéréotypé, expurgé de l'ambivalence et de la polyphonie, qui fondent la multiplicité complexe de profils individuels et de partage d'expériences. Il est étayé sur deux figures aussi immuables que leur antagonisme : la Victime et le Bourreau, l'assignation ethnique de l'un impliquant celle de l'autre. Cette figure publique d'une mémoire cultuelle, sur laquelle s'articulent des représentations spatialisées de la mythologie nationale hongroise, délimite ici clairement les frontières d'un « nous » minoritaire, en même temps que le socle sur laquelle émerge une nouvelle ethnicité politique.

Le cas des magyarophones de Transcarpathie est éclairant à ce sujet puisque les institutions issues de la mobilisation collective autour de l'ethnicité ont fini par devenir la colonne vertébrale des sociabilités dominantes. C'est par elles que tout l'espace social est innervé des deux réseaux politiques à forte hiérarchie interne.

Si le monde social magyarophone de Transcarpathie n'a pas réussi à résister à cette pression, c'est aussi parce que les élites qui en sont issues, en initiant la mobilisation, en 
instituant un espace politique particulier à l'ethnicité, ont défini elles-mêmes les normes, les vocables et les symboles de cette ethnicité. Comme le rappellent Tournon et Maiz (2005), les identités ethniques sont des ensembles malléables ; ainsi les élites hongroises ont modelé cette ethnicité en une image, susceptible de devenir un bien symbolique de valeur, économiquement et politiquement négociable sur les scènes politiques nationales instables et parfois rivales de la Hongrie et de l'Ukraine. Cette identité hongroise explicite et singulière de Transcarpathie n'a jamais existé jusqu'à ces dernières années, ni au sein de l'État hongrois d'avant 1920, ni dans la Transcarpathie soviétique : il n'existait qu'une ethnicité quotidienne, en contact permanent avec d'autres.

Pour autant, il ne s'agit pas d'affirmer que cette nouvelle ethnicité est une création « artificielle », montée de toute pièce, ni que c'est la mobilisation des années 1960 qui a construit la «magyarité » de Transcarpathie. Celle-ci préexistait et continue à exister sous des formes et dans des contextes différents. En revanche, c'est en effet le mouvement collectif qui politise cette ethnicité en la construisant sur le double pilier de la mémoire collective, spatialisée et ritualisée et de la langue, et en l'inscrivant dans l'espace public alors qu'elle demeurait dans le privé et le local jusqu'alors. Les élites, en surimposant ce nouvel espace public à une ethnicité quotidienne, latente jusque là, créent une l'ethnicité politique, publique (Tournon et Maiz 2005), organisée aujourd'hui en réseaux concurrents, qui prétend épouser l'ensemble des sociabilités des magyarophones. À partir du moment où l'ensemble de l'espace public est médiatisé par l'ethnicité, le renforcement de frontières culturelles qu'elle implique fait émerger une normativité appelant à l'isolement de la communauté.

Cependant, l'observation ethnographique du quotidien et de la vie religieuse des bourgs, villages et quartiers périphériques et l'accompagnement, souvent transfrontalier, de pratiques courantes permet de constater qu'une grande partie de l'économie et de l'organisation sociale locales de l'espace magyarophone de Transcarpathie est largement emboîtée dans celui d'autres groupes ethniques régionaux et transfrontaliers et mobilise des solidarités familiales et locales pré-existantes aux réseaux politiques polarisés. Si ces pratiques échappent donc à la logique politique de l'isolement ethnique, la normativité dominante de ce dernier renforce leur ancrage dans le domaine de l'informel. Illégales, selon les normes juridiques nationales, elles tendent à être perçues par l'élite ethnique comme illégitimes, polarisant ainsi le monde social local entre bénéficiaires de ressources économiques légitimées et travailleurs précaires, tentant de capter des ressources illégales.

Le tourisme - essentiellement de proximité - offre un cadre privilégié à la transformation locale des lieux et des ritualisations de la mémoire publique en biens dont la symbolique permet une marchandisation diversifiée. Légalisée ou informelle, cette activité permet de contourner le modèle ethniciste de légitimité politique et d'isolement, tout en capitalisant les ressources mémorielles créées par lui. Ainsi, pour le tourisme identitaire hongrois venant de Hongrie ou d'autres pays limitrophes ou occidentaux - les petits entrepreneurs locaux construisent une offre de visites, présentées - essentiellement sur internet - en termes d'apprentissage : revivre et partager l'expérience d'une magyarité sans frontières, alors que pour les visiteurs ukrainiens, roumains ou russes, les mêmes lieux seront présentés comme porteurs d'une altérité exotisante esthétisée à laquelle les nouvelles opportunités de voyage permettent enfin un accès aisé. Nature environnante intacte et bains thermaux en renaissance, échos de valeurs occidentales globalisées, constituent les points communs entre l'offre d'une 
expérience d'intimité culturelle, élargie dans le temps et l'espace, et celle, esthétique, d'une altérité proche.

Ainsi, au terme d'un itinéraire tortueux, la marchandisation touristique d'une mémoire ethnicisée, centrée sur la déportation, permet que celle-ci, de pièce maîtresse d'un " nous » collectif victimaire, opposé à d'autres et à celui des bourreaux, devienne objet de partages momentanés qui la dotent de multiples sens.

\section{Références citées}

Agulhon, Maurice, 1978. La « statuomanie » et l'histoire. Ethnologie française, 8 (mars-septembre), p. 145-72.

BALOG, Sándor, 1992, Sorsüldözöttek. Ungvár-Budapest : Galéria Kiadó.

Botlik, Jozsef, 1997, Harmas kereszt alatt. Budapest : Hatodik Sip Alapitvany Uj Mandatum Könyvkiado.

Brubaker, Rogers et alii, 2007. Nationalist Politics and Everyday Ethnicity in a Transylvanian Town. Princeton et Oxford : Princeton University Press.

BiBÓ, István, 1986. A kelet-európai kisállamok nyomorúsága (1946). In Bibó István : Válogatott tanulmányok II. kötet. Budapest : Magvetö, p. 185-265.

Collard, Anna, 1989. Investigating « Social Memory » in a Greek Context. In : History and ethnicity. Ed. by Elizabeth-McDonald Tonkin, Malcolm Maryon-Chapman. London : Routledge, p. 89-103.

DupKa György (éd.),

1993a. Egyetlen bünük magyarságuk volt. Emlékkönyv a sztálinizmus kárpálaljai áldozatairól (1944-1946). Ungvár-Budapest : Intermix.

1993b. « Sötét napok jöttek.. » Koncepciós perek magyar elitéltjeinek emlékkönyve 1944-1955. UngvárBudapest : Intermix.

ERIKSEN, Thomas Hylland, 1993. « Formal and informal nationalism », Ethnic and Racial Studies, 16 (January), p. $1-25$.

Fejös, Zoltàn, 1995. Kollektiv emlékezés és az etnikai identitàs megszerkesztése, Magyarságkutatás 1995-96. Szerk.: Diószegi László. Budapest : Teleki László Alapítvány, p. 125-141.

Hahn, Chris, Mihály SÁrkány et Peter Skalnik (éds), 2005. Studying peoples in the people's democracies. Socialist era anthropology in East-Central Europe. Munster : Lit Verlag.

Halbwachs, Maurice, 1994 [1925]. Les cadres sociaux de la mémoire. Paris : Albin Michel.

Herzfeld Michaël, 2007 [1997]. L'intimité culturelle. Politique sociale dans l'État-nation. Québec : Presses de l’Université Laval.

Hobsbawm, Eric J., 1992. « Ethnicity and Nationalism in Europe Today », Anthropology Today, 8. (February), p. 3-8.

Hofer, Tamás,,1992. Harc a rendszerváltásért szimbolikus mezőben. 1989. március 15-e Budapesten. Politikatudományi Szemle 1, p. 29-51.

Istenhez fohászkodva. Verses levelek, imák a sztálini lágerekben raboskodott magyarok verseiböl, imáiból, leveleiböl 1944-1957. Ungvár-Budapest : Intermix, 1992.

Karas, David, 2008. Politiques de l'ethnicité. Le cas des Hongrois de Transcarpathie. Mémoire de master en Politiques comparées, IEP, Paris.

Kertzer, David, 1988. Ritual, Politics, and Power. New Haven-London : Yale University Press.

Losonczy Anne-Marie, 1997. « Les itinéraires de la 'patrie'. De la construction de l'espace interpatriotique en Hongrie contemporaine ». Dire les autres. Réflexions et pratiques ethnologiques. Lausanne : Editions Payot, coll. Sciences humaines. 
Magocsi, Paul R. 1978. The Shaping of National Identity. Subcarpatian Rus', 1848-1948. Cambridge et Londres : Harvard University Press.

NAGY Jenö, 1992. Megaláztatásban. Ungvár-Budapest : Intermix.

PunYKó Mária, 1993. Reggelt adott az Isten. A szenvedés évei kárpátaljai népi elbeszélésekben. Debrecen : Györffy István Néprajzi Egyesület.

SzABó, László, 1993. Kárpátaljai demográfiai adatok. Ungvár-Budapest : Intermix.

SzEBENI Ilona, 1991. Merre van a magyar hazám ? Kényszermunkán a Szovjetunióban 1944-1949. Budapest : Széphalom Könyvmühely.

Tiz év a magyarsag szolgalataban, KMKSZ X. közgyûlése. Ungvar : 1999.

Tournon, Jean et Ramon Maiz (dir.), 2005. Ethnicisme et politique. Paris : L'Harmattan.

Wachtel, Nathan, 1986. Introduction. Special issue : Between Memory and History. History and Anthropology, 2, p. 207-224. 